Article

\title{
Antiphotoaging Effect of (2'S)-Columbianetin from Corydalis heterocarpa in UVA-Irradiated Human Dermal Fibroblasts
}

\author{
Jung Hwan Oh ${ }^{1}$, Fatih Karadeniz ${ }^{1}$, Jung Im Lee ${ }^{1}$, Hye Ran Kim ${ }^{2}$, Youngwan Seo ${ }^{3}$ and \\ Chang-Suk Kong $1,2, * \mathbb{D}$ \\ 1 Marine Biotechnology Center for Pharmaceuticals and Foods, College of Medical and Life Sciences, \\ Silla University, Busan 46958, Korea; wjdghks0171@naver.com (J.H.O.); karadenizf@outlook.com (F.K.); \\ think3433@daum.net (J.I.L.) \\ 2 Department of Food and Nutrition, College of Medical and Life Sciences, Silla University, Busan 46958, \\ Korea; gf5335@naver.com \\ 3 Department of Convergence Study on the Ocean Science and Technology, Ocean Science and Technology \\ School, Korea Maritime and Ocean University, Busan 49112, Korea; ywseo@kmou.ac.kr \\ * Correspondence: cskong@silla.ac.kr; Tel.: +82-51-999-5429
}

Received: 10 February 2020; Accepted: 1 April 2020; Published: 9 April 2020

\begin{abstract}
Ultraviolet A (UVA)-induced detrimental effects in the skin, also known as photoaging, are mediated with several pathways including oxidative stress generation and extracellular matrix (ECM) degradation. UVA irradiation results in excessive production of matrix metalloproteinases (MMPs), enzymes responsible for the degradation of ECM components such as collagen. In this study, the protective effects of $\left(2^{\prime} S\right)$-columbianetin against UVA-induced changes in matrix metalloproteinase-1 (MMP-1) and collagen production were investigated in human dermal fibroblasts (HDFs). The (2'S)-columbianetin was isolated from Corydalis heterocarpa. UVA exposure increased MMP-1 release from HDFs and diminished the release of type I pro-collagen. Treatment with $\left(2^{\prime} \mathrm{S}\right)$-columbianetin reversed these effects of UVA exposure. The (2'S)-columbianetin treatment also suppressed the intracellular expression of MMP-1 and increased type I pro-collagen expression. UVA exposure elevated the activation of p38, c-Jun-amino-terminal kinase (JNK) and extracellular signal-related kinase (ERK) as the mechanism to stimulate MMP-1 production. The (2'S)-columbianetin suppressed the phosphorylation of JNK and ERK. The (2'S)-columbianetin was also stimulated collagen production via TGF $\beta$ signaling cascade, relieving UVA-induced suppression of Smad2/3 phosphorylation and translocation. In conclusion, $\left(2^{\prime} S\right)$-columbianetin was suggested to be a potential cosmeceutical lead compound with antiphotoaging properties against UVA-induced collagen degradation.
\end{abstract}

Keywords: columbianetin; human dermal fibroblast; MAPK; photoaging; TGF $\beta$; UVA

\section{Introduction}

The dermis is the connective layer of the skin which connects the outermost epidermis layer with subcutaneous tissues. Although it is not directly exposed to environmental harmful effects, the penetration ability of ultraviolet (UV) rays causes several complications in the dermis layer upon long time exposure [1,2]. UVA is one of the light rays that are emitted by the sun together with UVB and UVC. UVB has the most detrimental and broad harmful effects and affects mainly the top layer of skin, causing sunburn, redness and cancer [3,4]. UVA irradiation can penetrate deep layers of skin and it is the main cause of premature skin aging and wrinkling [5]. UVA exposure increases the generation of reactive species in the dermis layer of the skin [6]. The UVA-mediated skin aging, also known 
as photoaging, arises due to the deleterious effects of UVA on the collagen and elastin backbone of the extracellular matrix (ECM) via matrix metalloproteinases (MMPs), natural ECM degraders $[7,8]$. UVA-induced generation of reactive species stimulates the expression and enzymatic activity of MMPs, especially MMP-1, -2 , and -9 which in turn causes the rapid digestion of collagen [9]. MMPs are crucially involved in the remodeling of ECM, migration of cells, and photoaging [10,11]. This cascade of stimulation leads to the damaged dermal ECM, which is the reason for the formation of wrinkles, and prematurely aged skin [12]. Like its UVB counterpart, UVA exposure is also closely linked with the activation of mitogen-activated protein kinase (MAPK) pathways [13]. MAPKs take part in MMP production and the cleavage of collagen in ECM $[14,15]$. Furthermore, the collagen synthesis pathways such as TGF $\beta /$ Smad are negatively affected by UVA exposure resulting in loss of elasticity in the skin [16].

Considering the role of MMPs in the harmful effects of UV irradiation, diseases and complications such as tumor metastasis, inflammation, atherosclerosis and cardiovascular diseases, numerous natural MMP inhibitors have been studied and developed [17,18]. A considerable portion of these MMP inhibitors was derived from phytochemicals such as polyphenols, flavonoids, coumarins, caffeic acids and their derivatives [19-21]. The (2'S)-columbianetin is a coumarin with several bioactivities including UVB-protective effects in keratinocytes [22]. In this context, the current study aimed to investigate the protective effects of $\left(2^{\prime} S\right)$-columbianetin against UVA induced damages in dermal fibroblasts, cells that are responsible for connective tissue formation which is a crucial step of the skin recovery. Therefore, as a part of the ongoing effect to develop cosmeceuticals against photoaging, (2'S)-columbianetin (Figure 1) was isolated from Corydalis heterocarpa, a salt marsh plant from Korea, and studied for its ability to revert detrimental effects of UVA on collagen and MMP mechanisms.

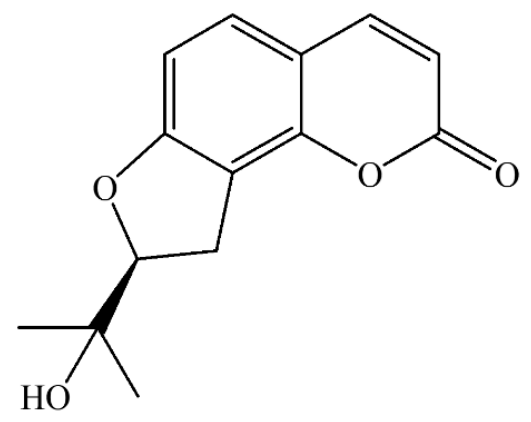

Figure 1. Chemical structure of $\left(2^{\prime} S\right)$-columbianetin.

\section{Results}

Prior to analysis of the potential UVA protective effects of ( $\left.2^{\prime} \mathrm{S}\right)$-columbianetin, its cytotoxicity was assessed by an 3-(4,5-dimethylthiazol-2-yl)-2,5-diphenyltetrazolium bromide (MTT) assay in human dermal fibroblast (HDF) cell line. Treatment with varying concentrations $(0,1,5,10,20$ and $25 \mu \mathrm{M})$ of $\left(2^{\prime} \mathrm{S}\right)$-columbianetin for $48 \mathrm{~h}$ showed a dose-dependent cytotoxicity in HDFs with an $\mathrm{IC}_{50}$ value of $93.89 \mu \mathrm{M}$ (Figure 2A). Cell viability was above $90 \%$ up to $10 \mu \mathrm{M}$, which was chosen as the highest dose of treatment. UVA irradiation was carried out at $10 \mathrm{~J} / \mathrm{cm}^{2}$ dose which was previously reported to be non-toxic for $24 \mathrm{~h}$ while inducing photoaging mechanisms (MMP-1 activation and inhibition of collagen synthesis) in HDFs [23]. Ahn et al. [22] reported that (2'S)-columbianetin showed a significant scavenging effect against UVB-mediated reactive oxygen species (ROS) generation in keratinocytes. In the current study, any scavenging effect of $\left(2^{\prime} S\right)$-columbianetin against UVA-mediated reactive species was investigated in human dermal fibroblasts by measuring nitrate amount in cell culture medium as a marker for reactive nitrogen species (NO) generation. UVA irradiation significantly increased the NO generation in HDFs (Figure 2B). The treatment with (2'S)-columbianetin dose-dependently decreased the UVA-induced NO generation suggesting that it might attenuate the UVA-induced reactive species generation in addition to reported protective effects against UVB. 
(A)

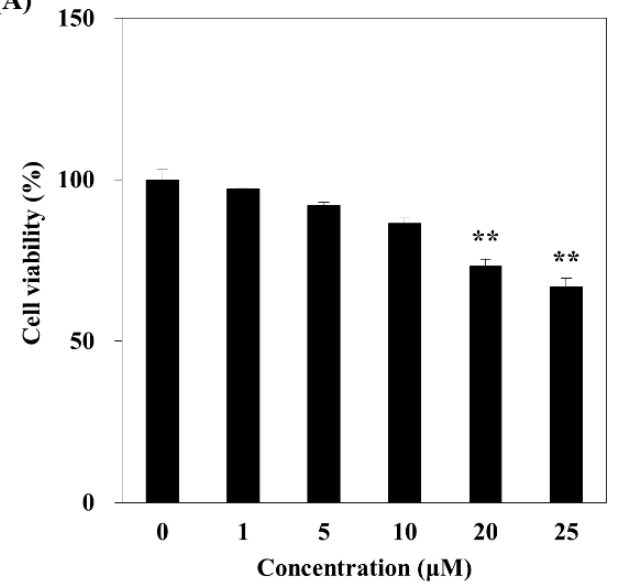

(B)

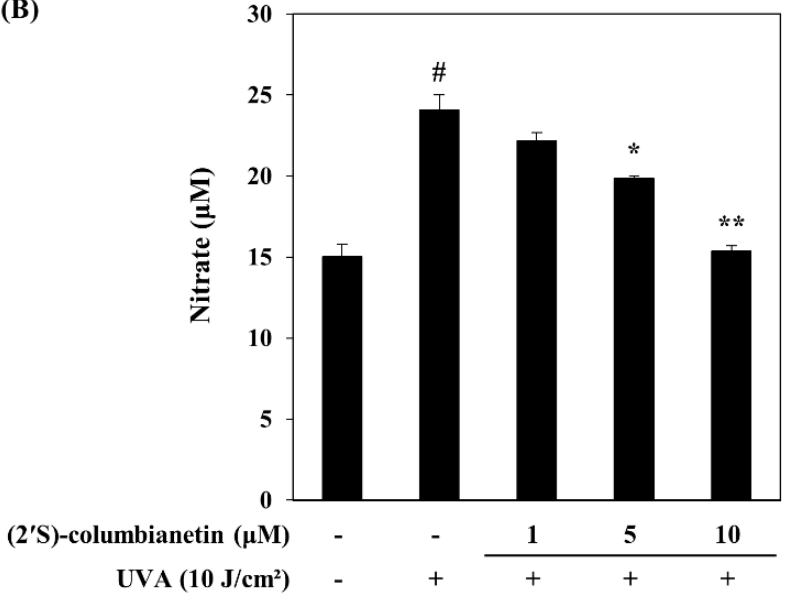

Figure 2. Effect of $\left(2^{\prime} S\right)$-columbianetin on viability of human dermal fibroblasts (HDFs) and nitrogen species (NO) generation in UVA-irradiated HDFs. (A) HDFs were incubated for $48 \mathrm{~h}$ following treatment with changing doses of $\left(2^{\prime} \mathrm{S}\right)$-columbianetin. Effects of $\left(2^{\prime} \mathrm{S}\right)$-columbianetin on the viability of HDFs was analyzed by 3-(4,5-dimethylthiazol-2-yl)-2,5-diphenyltetrazolium bromide (MTT) assay. Viability of the cells was calculated from absorbance values and given as the relative percentage of untreated control cells $(0 \mu \mathrm{M})$. Values are means $\pm \operatorname{SD}(n=3)$. ${ }^{* *} p<0.01$ compared to untreated control group $(0 \mu \mathrm{M})$. (B) HDFs were exposed to UVA radiation $\left(10 \mathrm{~J} / \mathrm{cm}^{2}\right)$ and treated with given concentrations of ( $\left.2^{\prime} \mathrm{S}\right)$-columbianetin. After $24 \mathrm{~h}$ incubation, nitric oxide generation was quantified as the nitrate amount in the cell culture medium. Values are means $\pm \operatorname{SD}(n=3)$. ${ }^{\#} p<0.01$ compared to non-irradiated blank group; ${ }^{*} p<0.05,{ }^{* *} p<0.01$ compared to UVA-irradiated untreated control group.

\subsection{Effect of (2'S)-Columbianetin on the UVA-Mediated Release of MMP-1 and Type I $\alpha 1$ Pro-Collagen}

The HDFs were exposed to UVA radiation $\left(10 \mathrm{~J} / \mathrm{cm}^{2}\right)$ with or without $(2$ S)-columbianetin $(1,5,10$ $\mu \mathrm{M})$ treatment. UVA-exposed cells released substantially increased levels of MMP-1 compared to the non-irradiated group (Figure 3A). The base MMP-1 level in HDF culture medium was 18,550 pg/mL and UVA exposure increased this amount to $20,849 \mathrm{pg} / \mathrm{mL}$. The presence of $\left(2^{\prime} \mathrm{S}\right)$-columbianetin suppressed the UVA-induced increase in MMP-1 levels in a concentration-dependent manner. The MMP-1 levels were reduced to $19,094 \mathrm{pg} / \mathrm{mL}$ after the $10 \mu \mathrm{M}\left(2^{\prime} \mathrm{S}\right)$-columbianetin treatment. The type $\mathrm{I} \alpha 1$ pro-collagen amount in UVA-exposed HDFs was significantly lower than non-irradiated group. The amount of type I $\alpha 1$ pro-collagen in irradiated HDF culture medium was dropped to $1753 \mathrm{pg} / \mathrm{mL}$ from $1934 \mathrm{pg} / \mathrm{mL}$ of non-irradiated HDFs. The ( 2 'S)-columbianetin treatment increased the type I $\alpha 1$ pro-collagen levels in irradiated HDF culture medium. At $10 \mu \mathrm{M}$ treatment group, type I $\alpha 1$ pro-collagen amount was raised to $1955 \mathrm{pg} / \mathrm{mL}$.

\subsection{Effect of (2'S)-Columbianetin on the UVA-Mediated Expression of MMP-1 and Collagen}

The expression levels of MMP-1 and type I pro-collagen in UVA-irradiated HDFs were analyzed at the gene and protein level. In addition, levels of collagen were also determined. UVA irradiation at a dose of $10 \mathrm{~J} / \mathrm{cm}^{2}$ notably increased the mRNA expression of MMP-1 in HDFs while decreasing expression of type I pro-collagen (Figure 4A). Parallel effects were observed in the protein levels of MMP-1 and type I pro-collagen (Figure 4B). However, UVA-induced changes in the MMP-1 and type I pro-collagen were ameliorated by $\left(2^{\prime} \mathrm{S}\right)$-columbianetin treatment in a concentration-dependent manner. UVA exposure resulted in decreased levels of collagen along with its precursor type I pro-collagen (Figure 4B). The collagen level was increased by the presence of $\left(2^{\prime} S\right)$-columbianetin in a similar fashion to that of type I pro-collagen. 
(A)

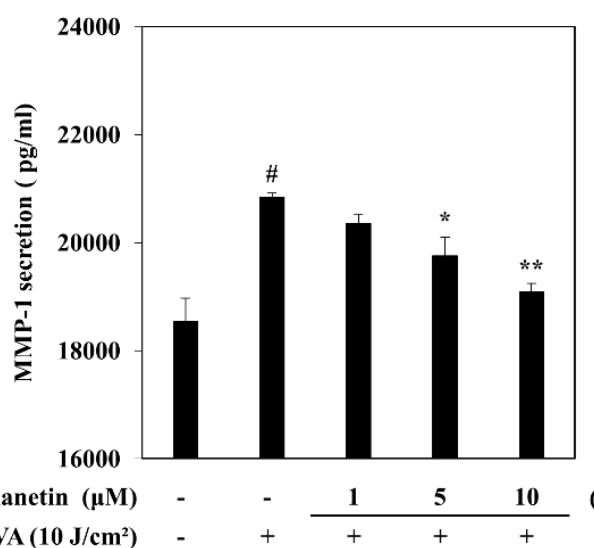

(B)

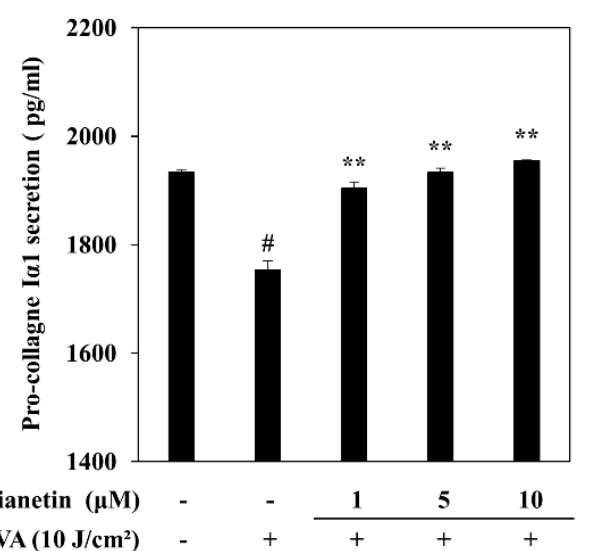

Figure 3. Effect of ( 2 'S)-columbianetin on the cellular release of MMP-1 (A) and type I $\alpha 1$ pro-collagen (B) in UVA-irradiated HDFs. The contents of MMP- 1 and type $\mathrm{I} \alpha 1$ pro-collagen were calculated from UVA-irradiated cell culture media following $24 \mathrm{~h}$ incubation with different concentrations of $\left(2^{\prime} \mathrm{S}\right)$-columbianetin using ELISA. Values are means $\pm \mathrm{SD}(n=3) .{ }^{*} p<0.01$ compared to non-irradiated blank group; ${ }^{*} p<0.05,{ }^{* *} p<0.01$ compared to UVA-irradiated untreated control group.

(A)

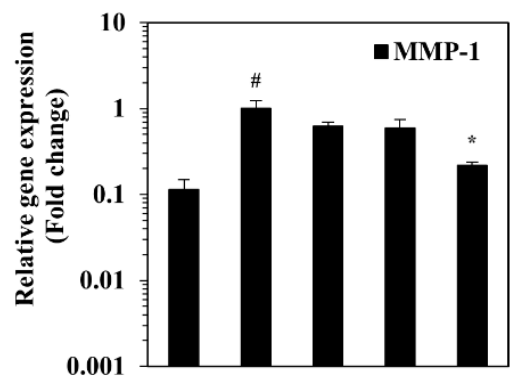

(2'S)-columbianetin $(\mu \mathrm{M})$ UVA $\left(10 \mathrm{~J} / \mathrm{cm}^{2}\right)-++++$

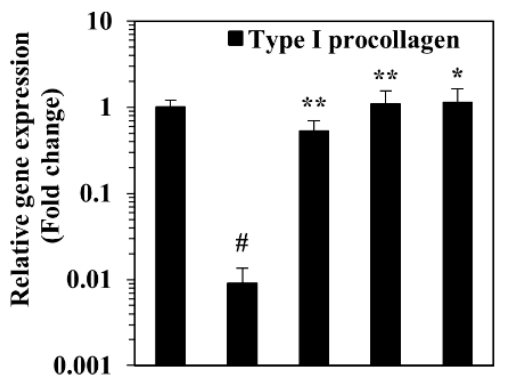

$\left(\mathbf{2}^{\prime} \mathrm{S}\right)$-columbianetin $(\mu \mathrm{M})$ UVA $\left(10 \mathrm{~J} / \mathrm{cm}^{2}\right)$
(B)
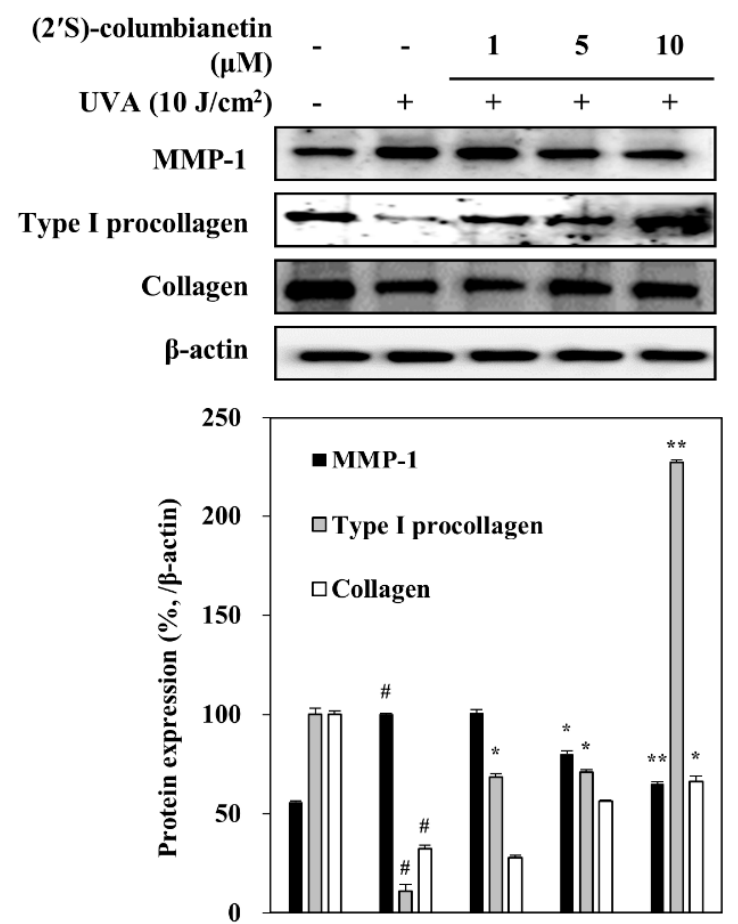

Figure 4. Effect of (2'S)-columbianetin on the mRNA (A) and protein (B) expression levels of the MMP-1 and type I $\alpha 1$ pro-collagen in UVA-irradiated HDFs after $24 \mathrm{~h}$ from treatment analyzed by Reverse Transcription-Quantitative Polymerase Chain Reaction (RT-qPCR) and Western blotting, respectively. $\beta$-actin was used as internal loading control. RT-qPCR expression quantity was shown relative to the untreated UVA-irradiated control, calculated by $2^{-(\Delta \Delta C t)}$ method. Western blot bands were densitometrically quantified and plotted as relative percentage of UVA-irradiated untreated control group. Values are means $\pm \mathrm{SD}(n=3) .{ }^{*} p<0.01$ compared to non-irradiated blank group; ${ }^{*} p<0.05$, ** $p<0.01$ compared to UVA-irradiated untreated control group. 


\subsection{Effect of (2'S)-Columbianetin on the UVA-Induced MAPK Activation}

The mitogen-activated kinase (MAPK) signaling cascade was investigated to determine whether ( $\left.2^{\prime} \mathrm{S}\right)$-columbianetin could intervene with the UVA-induced transcriptional activities of AP-1, MMP-1 expression inducing transcription factor. In this context, effects of $\left(2^{\prime} S\right)$-columbianetin on the total and phosphorylated (p-) levels of Jun-amino-terminal kinase (JNK), extracellular signal-related kinase (ERK1/2) and p38 MAPKs were investigated. The UVA-irradiated HDFs expressed significantly increased phosphorylation of JNK, ERK andp38 (Figure 5A). Treating irradiated HDFs with $10 \mu \mathrm{M}$ ( 2 'S)-columbianetin effectively suppressed the phosphorylation of ERK and JNK but failed to change the p-p38 levels significantly.

(A)
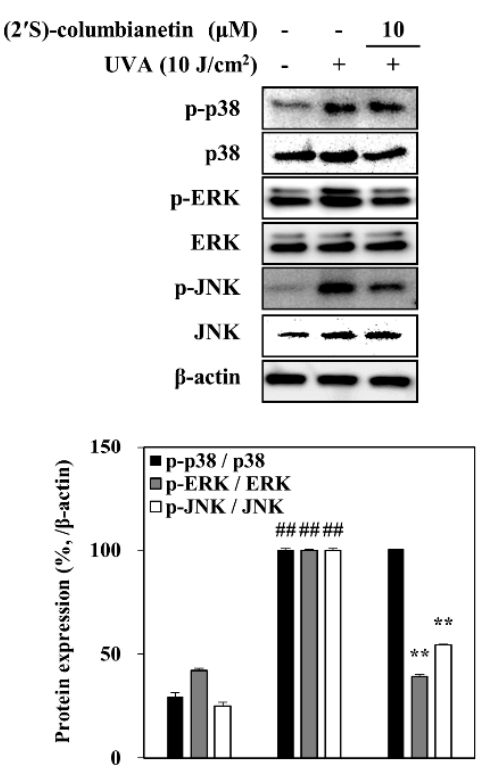

(2'S)-columbianetin $(\mu \mathrm{M})$ UVA $\left(10 \mathrm{~J} / \mathrm{cm}^{2}\right)$
(B)

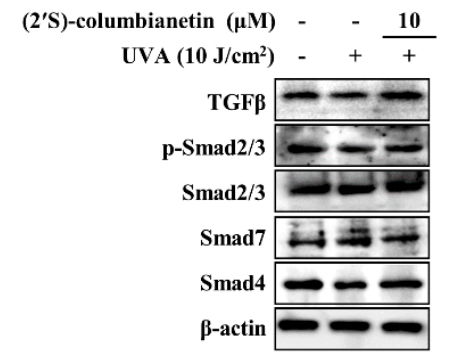

(2'S)-columbianetin $(\mu \mathrm{M})$

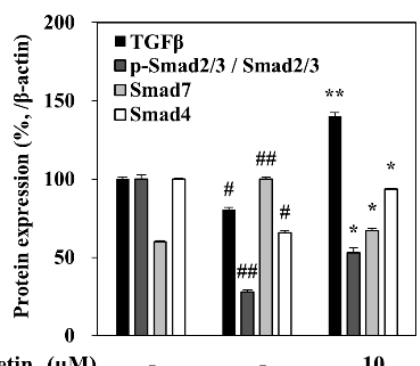

(C)

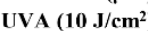

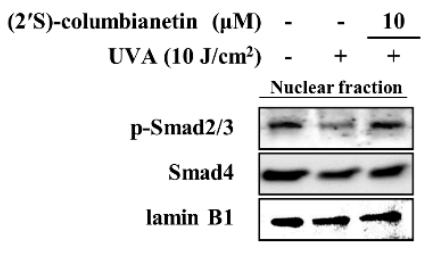

(2'S)-columbianetin (

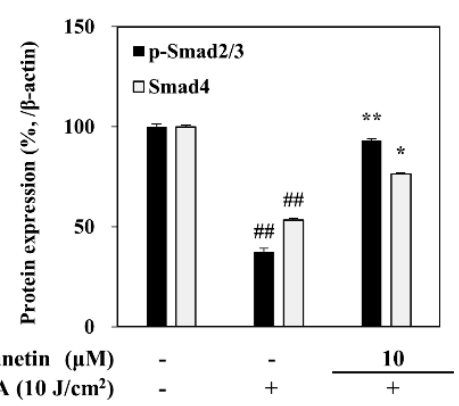

Figure 5. Effect of (2'S)-columbianetin on the protein levels of; total and phosphorylated (p-) p38, ERK and JNK MAPKs (A), TGF $\beta$, Smad2/3 (total and p-), Smad7 and Smad4 (B), and nuclear levels of p-Smad2/3 and Smad4 (C) in UVA irradiated HDFs following $24 \mathrm{~h}$ treatment. Protein levels were observed by Western blotting. Bands were quantified densitometrically and shown as relative percentage of UVA-irradiated untreated control group. $\beta$-actin and lamin B1 were used as internal loading control. Values are means $\pm \mathrm{SD}(n=3) .{ }^{\#} p<0.05,{ }^{\# \#} p<0.01$ compared to non-irradiated blank group; ${ }^{*} p<0.05,{ }^{* *} p<0.01$ compared to UVA-irradiated untreated control group.

\subsection{Effect of (2'S)-Columbianetin on the UVA-Induced Suppression of TGF $\beta$ Pathway}

To elucidate the possible mechanisms behind the stimulatory effects of $\left(2^{\prime} \mathrm{S}\right)$-columbianetin on pro-collagen and collagen levels, changes in the TGF $\beta$ signaling were investigated via analyzing the levels of TGF $\beta$ and Smad phosphorylation. As seen in Figure 5B, UVA exposure $\left(10 \mathrm{~J} / \mathrm{cm}^{2}\right)$ caused a significant decrease in the TGF $\beta$ levels. In addition, the downstream activators of TGF $\beta, p-S m a d 2 / 3$ and Smad4 levels were also similarly decreased. The level of Smad7, which is the negative regulator of the TGF $\beta$ cascade, was increased following UVA irradiation. The presence of $\left(2^{\prime} S\right)$-columbianetin $(10 \mu \mathrm{M})$ reversed the effects of UVA on TGF $\beta$ pathway by increasing the $\mathrm{p}$-Smad2/3 levels. Likewise, Smad4 protein level was also increased by $\left(2^{\prime} \mathrm{S}\right)$-columbianetin $(10 \mu \mathrm{M})$ treatment while Smad7 protein level was concurrently decreased. The effect of ( 2 'S)-columbianetin on $\mathrm{p}-\mathrm{Smad} 2 / 3 / 4$ complex-dependent synthesis of collagen was further analyzed by levels of $\mathrm{p}-\mathrm{Smad} 2 / 3$ and Smad 4 in nuclear fractions of 
the HDFs. UVA exposure resulted in decreased levels of $\mathrm{p}-\mathrm{Smad} 2 / 3$ and Smad4 in nuclear fractions which was similarly increased by the treatment with $\left(2^{\prime} \mathrm{S}\right)$-columbianetin $(10 \mu \mathrm{M})$ (Figure $\left.5 \mathrm{C}\right)$.

\section{Discussion}

In the current study, (2'S)-columbianetin, a bioactive coumarin isolated from $C$. heterocarpa, was studied for its anti-photoaging properties in UVA irradiated dermal fibroblasts. The ( 2 'S)-columbianetin was shown to possess important bioactivities which include protective effects against UVB-induced damages in keratinocytes, cells forming the upper epidermis layer of skin, via antioxidant and MMP-1 inhibitory activities [22]. In order to confirm its photoprotective effects against UVA irradiation in dermal fibroblasts, the present study investigated the effects of (2'S)-columbianetin on UVA-irradiated changes in MMP-1 and collagen production using human dermal fibroblast cell line.

UVA rays can penetrate deeper than UVB until the bottom layers of the dermis [5]. Subtoxic UVA exposure induced several detrimental effects in the dermis such as premature skin aging, also known as photoaging [7]. Cells exposed to UVA expressed increasing amounts of MMPs. MMPs are key enzymes in ECM building and maintenance due to their ability to cleave main ECM components such as collagen. MMP- 1 is a member of the collagenase subfamily of MMPs and responsible for the breakdown of ECM during regular cellular processes such as tissue remodeling and recovery as well as diseases such as metastasis by initially cleaving the type I, II and III collagen [24,25]. However, UVA irradiation upregulates the MMP-1 production and subsequently reduces the collagen synthesis in the dermal fibroblasts. The main outcome of these changes is loss of skin elasticity and strength $[7,8]$. It was shown in Figure 3 that UVA exposure increased the MMP-1 production and decreased the type I $\alpha 1$ pro-collagen. Treating dermal fibroblasts with $10 \mu \mathrm{M}\left(2^{\prime} \mathrm{S}\right)$-columbianetin notably ameliorated the effects of UVA irradiation by increasing the type I $\alpha 1$ pro-collagen production which is the precursor of type I collagen and decreasing the MMP-1 release (Figure 3). This was further evidenced by analyzing its effects on mRNA and protein expression levels of MMP-1 and type I pro-collagen. The ( $\left.2^{\prime} \mathrm{S}\right)$-columbianetin reversed the effects of UVA on mRNA and protein levels: increasing pro-collagen expression while suppressing MMP-1 (Figure 4).

It was reported that UVA irradiation upregulated the production of MMP-1 through activation of MAPKs via cell surface cytokine and stress receptors due to elevated oxidative stress [26]. UVA-induced activation of ERK, JNK and p38 MAPKs further activates the AP-1 transcription factor which stimulates the transcriptional expression of MMP genes [26,27]. The overexpression of MMPs in fibroblasts causes ECM to lose its integrity. As seen in Figure 5A, UVA irradiation significantly increased the phosphorylation of p38, ERK and JNK in HDFs. The $\left(2^{\prime} \mathrm{S}\right)$-columbianetin $(10 \mu \mathrm{M})$ treatment slightly decreased the ERK activation while significantly suppressing the activation of JNK. On the other hand, it did not affect p38 phosphorylation. Results indicated that (2'S)-columbianetin downregulated the UVA-induced MMP-1 production through a JNK-mediated signaling which speculatively resulted in decreased AP-1 activity.

The collagen synthesis in dermal fibroblasts is partly regulated by TGF $\beta$ and Smad cascades [28]. Stimulated TGF $\beta$ receptor facilitates the phosphorylation of Smad2 and Smad3 which together form an active complex with Smad4. The Smad2/3/4 complex then initiates the type I pro-collagen expression after its nuclear translocation. This cascade is negatively regulated by Smad7 protein which inhibits the formation of active Smad2/3/4 complex [28,29]. UVA exposure caused a decrease in the both production of TGF $\beta$ cytokine and phosphorylation of Smad2/3 complex (Figure 5B). As expected, this was accompanied by an increase in Smad7 level. Presence of $10 \mu \mathrm{M}\left(2^{\prime} \mathrm{S}\right)$-columbianetin relieved the suppression on p-Smad2/3 levels. Likewise, Smad7 levels were decreased by (2S)-columbianetin treatment. Effect of $\left(2^{\prime} S\right)$-columbianetin on the Smad2/3-mediated collagen synthesis was further investigated in the nuclear fractions of UVA-irradiated HDFs. UVA-mediated decrease in the levels of p-Smad $2 / 3$ and Smad 4 in the nucleus was countered by $10 \mu \mathrm{M}$ of $\left(2^{\prime} S\right)$-columbianetin which strongly increased the nuclear levels of p-Smad2/3 (Figure 5C). 
In conclusion, $\left(2^{\prime} S\right)$-columbianetin from $C$. heterocarpa exhibited protective properties against UVA-induced photoaging in human dermal fibroblasts. Treatment with ( $\left.2^{\prime} \mathrm{S}\right)$-columbianetin suppressed the UVA-induced MMP-1 overexpression through inhibition of MAPK phosphorylation. Furthermore, it ameliorated UVA-induced decrease in collagen synthesis by stimulating the activation of TGF $\beta /$ Smad cascade. Taken together, $\left(2^{\prime} \mathrm{S}\right)$-columbianetin is a potential candidate to be employed as a natural cosmeceutical against UVA-induced photoaging.

\section{Materials and Methods}

\subsection{Isolation of (2'S)-Columbianetin}

C. heterocarpa plants were collected in Muando, Jeollanamdo, Korea during July 2018. Leaves, stems and roots of plants were washed, chopped, ground and extracted using $\mathrm{MeOH}(31 \times 2)$ and $\mathrm{CH}_{2} \mathrm{Cl}_{2}(3 \mathrm{l} \times 2)$, respectively. Each extraction process lasted 2 days. The combined crude extracts $(20.5 \mathrm{~g})$ were dried in vacuo and later separated between $n$-hexane $(3.6 \mathrm{~g}), 85 \%$ aqueous (aq.) $\mathrm{MeOH}$ $(6.0 \mathrm{~g})$ and the remaining residue was further separated between $n-\mathrm{BuOH}(2.2 \mathrm{~g})$ and water $(10.0 \mathrm{~g})$. The $\left(2^{\prime} \mathrm{S}\right)$-columbianetin $(326.1 \mathrm{mg})$ was isolated from a portion of the $85 \%$ aq. $\mathrm{MeOH}$ fraction [22].

\subsection{Human Dermal Fibroblast (HDF) Culture and Cytotoxicity Assay}

HDF cells (C-12302; PromoCell, obtained from Heidelberg, Germany) were cultured in Fibroblast Growth Medium (C-23020, PromoCell) and kept in $37^{\circ} \mathrm{C}$ incubators with a humidified $(90 \%$ relative humidity) atmosphere containing $5 \% \mathrm{CO}_{2}$ between the experiments.

Any possible toxic effect of (2'S)-columbianetin in cells was investigated by colorimetric MTT assay as previously described [22]. Briefly, HDFs were seeded in 96-well plates $\left(1 \times 10^{3} \mathrm{cell} /\right.$ well $)$ and treated with $1,5,10,20$ and $25 \mu \mathrm{M}\left(2^{\prime} S\right)$-columbianetin along with untreated control group following $>90 \%$ confluence. Culture medium was replaced with $100 \mu \mathrm{L}$ MTT solution $(1 \mathrm{mg} / \mathrm{mL})$ following a 2-day incubation. The plate was then kept in dark at $37^{\circ} \mathrm{C}$ for $4 \mathrm{~h}$. Wells were aspired, and cells were washed with phosphate buffer saline (PBS). Ten microliters of $100 \%$ DMSO were introduced to each well to dissolve the formazan crystals and absorbance values of wells at $540 \mathrm{~nm}$ were measured using GENios FL microplate reader (Tecan Austria GmbH, Grodig, Austria). Changes in the viability of cells were calculated as a percentage of the untreated blank group which was considered to be $100 \%$ alive and compared to each concentration of (2'S)-columbianetin treatment.

\subsection{UVA Irradiation}

HDFs were irradiated by UVA using a Bio-Sun UV Irradiation System (Vilber Lourmat, Marine, France) fitted with a UVA source designed for microplates. HDFs grown in microplates were irradiated at $10 \mathrm{~J} / \mathrm{cm}^{2}$ UVA dose. Cells were irradiated in phosphate-buffered saline (PBS) without the plastic lid. When the irradiation received matched the desired programmed energy, the UVA irradiation stopped automatically, and subsequently the cells were incubated with DMEM without FBS until analysis.

\subsection{Enzyme-Linked Immunosorbent Assay (ELISA)}

Release of MMP- 1 and type I $\alpha 1$ procollagen in UVA-irradiated HDFs was analyzed by ELISA. Cells $\left(1 \times 10^{6}\right.$ cell/well $)$ were pre-incubated in 6-well plates for $24 \mathrm{~h}$ to reach $>90 \%$ confluence and washed with PBS prior to UVA $\left(10 \mathrm{~J} / \mathrm{cm}^{2}\right)$ exposure. Following UVA irradiation, the cells were treated with or without different concentrations $(1,5,10 \mu \mathrm{M})$ of $\left(2^{\prime} \mathrm{S}\right)$-columbianetin for $24 \mathrm{~h}$. Cell culture medium from each well were analyzed for its MMP- 1 and type I $\alpha 1$ procollagen contents per manufacturer's instructions of the ELISA kit (R\&D systems, Inc., Minneapolis, MN, USA).

\subsection{Reverse Transcription-Quantitative Polymerase Chain Reaction (RT-qPCR) Analysis}

Cells $\left(1 \times 10^{6}\right.$ cell/well $)$ were grown to $>90 \%$ confluence prior to UVA irradiation. Following UVA irradiation cells were treated with or without $\left(2^{\prime} \mathrm{S}\right)$-columbianetin for $24 \mathrm{~h}$. Total RNA was isolated 
from nonirradiated and irradiated (UVA, $10 \mathrm{~J} / \mathrm{cm}^{2}$ ) HDFs using AccuPrep ${ }^{\circledR}$ Universal RNA Extraction Kit (Bioneer, Daejeon, Korea) according to manufacturer's instructions. Total RNA was treated with RNase-free DNase I (Thermo Fisher Scientific, Rockford, IL, USA). The cDNA synthesis from total RNA was performed using CellScript All-in-One cDNA synthesis Master Mix (CellSafe, Yongin, Korea) following the producer's directions. cDNA amplification was carried out with quantitative PCR in a Thermal Cycler Dice ${ }^{\circledR}$ Real Time System TP800 (Takara Bio Inc., Ohtsu, Japan) following the manufacturer's protocol. Briefly $1.0 \mu \mathrm{L}$ of cDNA sample and $10.5 \mu \mathrm{L}$ of Luna ${ }^{\circledR}$ Universal qPCR Mix (New England Biolabs, Ipswich, MA, USA) were mixed with forward and reverse primers in nuclease-free water. The target cDNA was amplified using following forward and reverse primers; MMP-1, forward 5'-TCT GAC GTT GAT CCC AGA GAG CAG-3', reverse 5'-CAG GGT GAC ACC AGT GAC TGC AC-3'; type I procollagen, forward 5'-CTC GAG GTG GAC ACC ACC CT-3', reverse $5^{\prime}$-CAG CTG GAT GGC CAC ATC GG-3'; $\beta$-actin, forward $5^{\prime}$-AGA TCA AGA TCA TTG CTC CTC CTG-3', reverse 5'-CAA GAA AGG GTG TAA CGC AAC TAA G-3'. The PCR amplification was carried out with an initial denaturation at $95^{\circ} \mathrm{C}$ for $1 \mathrm{~min}$, followed by $40 \mathrm{PCR}$ cycles, each cycle consisting of $95^{\circ} \mathrm{C}$ for $15 \mathrm{~s}$ and $60^{\circ} \mathrm{C}$ for $30 \mathrm{~s}$. Relative quantification was calculated using the $2^{-(\Delta \Delta C \mathrm{CT})}$ method. $\beta$-Actin was used as an internal control.

\subsection{Western Blotting}

Protein levels were investigated using immunoblotting according to common Western blotting protocols. HDFs cultured in 6-well plates were treated with or without $\left(2^{\prime} \mathrm{S}\right)$-columbianetin $(1,5,10 \mu \mathrm{M})$ and exposed to UVA $\left(10 \mathrm{~J} / \mathrm{cm}^{2}\right)$ irradiation prior to $48 \mathrm{~h}$ incubation. Following incubation, wells were aspired and cells were lysed by vigorous pipetting in $1 \mathrm{~mL}$ of RIPA buffer (Sigma-Aldrich, St. Louis, $\mathrm{MO}, \mathrm{USA}$ ) at $4{ }^{\circ} \mathrm{C}$. Protein content of the lysates was measured with a bicinchoninic acid (BCA) protein assay kit (Thermo Fisher Scientific) following kit's protocol. Same amount $(20 \mu \mathrm{g})$ of protein from each well was loaded onto $12 \%$ SDS-polyacrylamide gel and run at $100 \mathrm{~V}$. Proteins on gel were then transferred onto a polyvinylidene fluoride membrane (Amersham; GE Healthcare, Little Chalfont, UK) using a wet system run at $100 \mathrm{~V}$ for $1 \mathrm{~h}$ at $4{ }^{\circ} \mathrm{C}$. Membranes were then incubated for $1 \mathrm{~h}$ at room temperature in 5\% skimmed milk for blocking. Blocked membranes were washed with 1X TBST and incubated with primary antibodies (diluted 1:1000) against MMP-1 (sc-6837; Santa Cruz Biotechnology, Santa Cruz, CA, USA), type I pro-collagen (sc-8782; Santa Cruz Biotechnology), collagen (sc-29318; Santa Cruz Biotechnology), p38 (\#8690; Cell Signaling Technology, Beverly, MA, USA), p-p38 (\#4511; Cell Signaling Technology), JNK (LF-PA0047; Thermo Fisher Scientific), p-JNK (sc-293136; Santa Cruz Biotechnology), ERK (\#4695; Cell Signaling Technology), p-ERK (\#4370; Cell Signaling Technology), TGF $\beta$ (\#3711; Cell Signaling Technology), Smad2/3 (sc-133098; Santa Cruz Biotechnology), p-Smad2/3 (sc-11769; Santa Cruz Biotechnology), Smad7 (sc-101152; Santa Cruz Biotechnology), Samd4 (sc-56479; Santa Cruz Biotechnology), lamin B1 (sc-374015; Santa Cruz Biotechnology) and $\beta$-actin (sc-47778; Santa Cruz Biotechnology) in primary antibody dilution buffer containing 1X TBST with 5\% bovine serum albumin overnight at $4{ }^{\circ} \mathrm{C}$. Membranes were then incubated with horseradish-peroxidase-conjugated secondary antibodies (diluted 1:1000) specific to the primary antibody source (anti-mouse: \#7076, anti-rabbit: \#7074; Cell Signaling Technology) at room temperature for $1 \mathrm{~h}$. Detection of proteins on blotted membranes was achieved using a ECL Western blot detection kit (Amersham) according to the manufacturer's instructions. Protein bands were imaged with CAS-400SM Davinch-Chemi imager ${ }^{\mathrm{TM}}$ (Davinch-K).

\subsection{Statistical Analysis}

All numerical data were given as the mean \pm standard deviation. Statistical differences between the means of the sample groups were calculated by the analysis of variance (ANOVA) followed by Duncan's multiple range test using SAS v9.1 software (SAS Institute, Cary, NC, USA). Any statistically significant difference between the groups were determined at the $p<0.05$ and $p<0.01$ levels. 
Author Contributions: All authors have read and agreed to the published version of the manuscript. Conceptualization and methodology, F.K., J.H.O. and Y.S.; validation, formal analysis and investigation, J.H.O., H.R.K. and J.I.L.; resources, C.-S.K.; data curation, F.K., C.-S.K. and Y.S.; writing-original draft preparation, visualization, F.K.; supervision, and project administration, C.S.K. and Y.S.; funding acquisition, C.S.K.

Funding: This research was a part of the project titled 'Development of cosmeceutical materials from Corydalis heterocarpa and Limonium tetragonum through optimizing extraction and isolation of bioactive ingredients', funded by the Ministry of Oceans and Fisheries, Korea. This work was supported by the BB21+ project in 2019.

Conflicts of Interest: The authors declare no conflict of interest.

\section{References}

1. Marionnet, C.; Tricaud, C.; Bernerd, F. Exposure to non-extreme solar UV daylight: Spectral characterization, effects on skin and photoprotection. Int. J. Mol. Sci. 2014, 16, 68-90. [CrossRef] [PubMed]

2. Krutmann, J.; Bouloc, A.; Sore, G.; Bernard, B.A.; Passeron, T. The skin aging exposome. J. Dermatol. Sci. 2017, 85, 152-161. [CrossRef] [PubMed]

3. Ichihashi, M.; Ueda, M.; Budiyanto, A.; Bito, T.; Oka, M.; Fukunaga, M.; Tsuru, K.; Horikawa, T. UV-induced skin damage. Toxicology 2003, 189, 21-39. [CrossRef]

4. Lee, J.H.; An, H.T.; Chung, J.H.; Kim, K.H.; Eun, H.C.; Cho, K.H. Acute effects of UVB radiation on the proliferation and differentiation of keratinocytes. Photodermatol. Photoimmunol. Photomed. 2002, 18, $253-261$. [CrossRef]

5. Krutmann, J. Ultraviolet A radiation-induced biological effects in human skin: Relevance for photoaging and photodermatosis. J. Dermatol. Sci. 2000, 23, S22-S26. [CrossRef]

6. Vile, G.F.; Tyrrell, R.M. Uva radiation-induced oxidative damage to lipids and proteins in vitro and in human skin fibroblasts is dependent on iron and singlet oxygen. Free Radic. Biol. Med. 1995, 18, 721-730. [CrossRef]

7. $\mathrm{Xu}, \mathrm{Y}$; Fisher, G.J. Ultraviolet (UV) light irradiation induced signal transduction in skin photoaging. J. Dermatol. Sci. Suppl. 2005, 1, S1-S8. [CrossRef]

8. Dong, K.K.; Damaghi, N.; Picart, S.D.; Markova, N.G.; Obayashi, K.; Okano, Y.; Masaki, H.; Grether-Beck, S.; Krutmann, J.; Smiles, K.A.; et al. UV-induced DNA damage initiates release of MMP-1 in human skin. Exp. Dermatol. 2008, 17, 1037-1044. [CrossRef]

9. Rittié, L.; Fisher, G.J. UV-light-induced signal cascades and skin aging. Ageing Res. Rev. 2002, 1, 705-720. [CrossRef]

10. Rohani, M.G.; Parks, W.C. Matrix remodeling by MMPs during wound repair. Matrix Biol. 2015, 44-46, 113-121. [CrossRef]

11. Wlaschek, M.; Tantcheva-Poór, I.; Naderi, L.; Ma, W.; Schneider, L.A.; Razi-Wolf, Z.; Schüller, J.; Scharffetter-Kochanek, K. Solar UV irradiation and dermal photoaging. J. Photochem. Photobiol. B Biol. 2001, 63, 41-51. [CrossRef]

12. Quan, T.; Qin, Z.; Xia, W.; Shao, Y.; Voorhees, J.J.; Fisher, G.J. Matrix-degrading metalloproteinases in photoaging. J. Investig. Dermatol. Symp. Proc. 2009, 14, 20-24. [CrossRef] [PubMed]

13. Bode, A.M.; Dong, Z. Mitogen-activated protein kinase activation in UV-induced signal transduction. Sci. STKE 2003, 167, re2:1-re2:15. [CrossRef] [PubMed]

14. Reunanen, N.; Li, S.P.; Ahonen, M.; Foschi, M.; Han, J.; Kähäri, V.M. Activation of p38 $\alpha$ MAPK enhances collagenase-1 (matrix metalloproteinase (MMP)-1) and stromelysin-1 (MMP-3) expression by mRNA stabilization. J. Biol. Chem. 2002, 277, 32360-32368. [CrossRef]

15. Panich, U.; Chaiprasongsuk, A.; Lohakul, J.; Soontrapa, K.; Sampattavanich, S. Photoprotective role of Nrf2 in UVA-mediated MMP-1 via MAPK/AP-1 signaling in keratinocyte HaCaT cells and mouse skin: The photoprotective effects of sulforaphane. Free Radic. Biol. Med. 2016, 100, S44. [CrossRef]

16. Gambichler, T.; Skrygan, M.; Tomi, N.S.; Breuksch, S.; Altmeyer, P.; Kreuter, A. Significant downregulation of transforming growth factor- $\beta$ signal transducers in human skin following ultraviolet-A1 irradiation. Br. J. Dermatol. 2007, 156, 951-956. [CrossRef]

17. Cathcart, J.M.; Cao, J. MMP inhibitors: Past, present and future. Front. Biosci. 2015, 20, 1164-1178.

18. Mannello, F. Natural bio-drugs as matrix metalloproteinase inhibitors: New perspectives on the horizon? Recent Pat. Anticancer Drug Discov. 2008, 1, 91-103. [CrossRef]

19. Shu, L.; Cheung, K.L.; Khor, T.O.; Chen, C.; Kong, A.N. Phytochemicals: Cancer chemoprevention and suppression of tumor onset and metastasis. Cancer Metastasis Rev. 2010, 29, 483-502. [CrossRef] 
20. Tan, W.; Lu, J.; Huang, M.; Li, Y.; Chen, M.; Wu, G.; Gong, J.; Zhong, Z.; Xu, Z.; Dang, Y.; et al. Anti-cancer natural products isolated from Chinese medicinal herbs. Chin. Med. 2011, 6, 27. [CrossRef]

21. Kimura, Y. Antitumor and antimetastatic actions of various natural products. Stud. Nat. Prod. Chem. 2008, 34, 35-76.

22. Ahn, B.N.; Kim, J.A.; Kong, C.S.; Seo, Y.; Kim, S.K. Protective effect of (2'S)-columbianetin from corydalis heterocarpa on UVB-induced keratinocyte damage. J. Photochem. Photobiol. B Biol. 2012, 109, $20-27$. [CrossRef] [PubMed]

23. Oh, J.H.; Karadeniz, F.; Lee, J.I.; Park, S.Y.; Seo, Y.; Kong, C.S. Anticatabolic and anti-inflammatory effects of myricetin 3-O- $\beta$-D-galactopyranoside in UVA-irradiated dermal cells via repression of MAPK/AP-1 and activation of TGF $\beta /$ Smad. Molecules 2020, 25, 1331. [CrossRef]

24. Pardo, A.; Selman, M. MMP-1: The elder of the family. Int. J. Biochem. Cell Biol. 2005, 37, 283-288. [CrossRef] [PubMed]

25. Conlon, G.A.; Murray, G.I. Recent advances in understanding the roles of matrix metalloproteinases in tumour invasion and metastasis. J. Pathol. 2019, 247, 629-640. [CrossRef] [PubMed]

26. Park, C.H.; Min, J.L.; Ahn, J.; Kim, S.; Hyeon, H.K.; Kyu, H.K.; Hee, C.E.; Jin, H.C. Heat shock-induced matrix metalloproteinase (MMP)-1 and MMP-3 are mediated through ERK and JNK activation and via an autocrine interleukin-6 loop. J. Investig. Dermatol. 2004, 123, 1012-1019. [CrossRef] [PubMed]

27. Klotz, L.-O.; Pellieux, C.; Briviba, K.; Pierlot, C.; Aubry, J.-M.; Sies, H. Mitogen-activated protein kinase (p38-, JNK-, ERK-) activation pattern induced by extracellular and intracellular singlet oxygen and UVA. Eur. J. Biochem. 1999, 260, 917-922. [CrossRef]

28. Verrecchia, F.; Mauviel, A. Control of connective tissue gene expression by TGF beta: Role of Smad proteins in fibrosis. Curr. Rheumatol. Rep. 2002, 4, 143-149. [CrossRef]

29. Yan, X.; Liao, H.; Cheng, M.; Shi, X.; Lin, X.; Feng, X.H.; Chen, Y.G. Smad7 protein interacts with receptor-regulated Smads (R-Smads) to inhibit transforming growth factor- $\beta$ (TGF- $\beta$ )/Smad signaling. J. Biol. Chem. 2016, 291, 382-392. [CrossRef]

(C) 2020 by the authors. Licensee MDPI, Basel, Switzerland. This article is an open access article distributed under the terms and conditions of the Creative Commons Attribution (CC BY) license (http://creativecommons.org/licenses/by/4.0/). 